\title{
Enterovirus-related diarrhoea in Guangdong, China: clinical features and implications in hand, foot and mouth disease and herpangina
}

Hong-Tao Zhou ${ }^{1,3 *}$, Hai-Su Yi ${ }^{1}$, Yong-Hui Guo ${ }^{1}$, Yu-Xian Pan', Shao-Hua Tao ${ }^{2}$, Bin Wang ${ }^{2}$, Man-Jun Chen ${ }^{1}$, Mei Yang ${ }^{4}$ and Nan $\mathrm{Yu}^{1 *}$

\begin{abstract}
Background: A series of complications caused by enteroviruses, including meningitis, encephalitis, acute flaccid paralysis, acute cardiopulmonary failure, respiratory infection, and myocardial injury have been reported in hand, foot and mouth disease/herpangina (HFMD/HA). However, the complication of diarrhoea caused by enteroviruses has been neglected, and a summary of its clinical features and impact on HFMD/HA is unavailable.

Methods: We included inpatients with HFMD/HA admitted to the Paediatric Department of Zhujiang Hospital during 2009-2012. We summarised and compared clinical data for cases with and without diarrhoea, and determined enterovirus serotypes by reverse transcriptase polymerase chain reaction and genotyping based on a partial-length fragment of viral protein 1 or the $5^{\prime}$-untranslated region.

Results: There were 804 inpatients with HFMD/HA and 28 (3.5\%) presented with diarrhoea. Gastrointestinal symptoms were mild in most cases of diarrhoea (82.1\%), with high prevalence of no dehydration (82.1\%), short duration of diarrhoea (78.6 \%) and watery stools (75.0 \%). The prevalence of multi-organ dysfunction syndrome (10.7 vs $0.40 \%)(p=0.001)$, hepatic injury (14.3 vs $3.4 \%)(p=0.019)$, myocardial injury (21.4 vs $6.1 \%)$ $(p=0.002)$ and convulsion ( 21.4 vs $7.2 \%)(p=0.016)$ was significantly higher in the diarrhoea than no diarrhoea group. There was no significant difference between the two groups regarding prevalence of death, altered consciousness, paralysis, central nervous system involvement, or acute respiratory infection.

Conclusions: Most patients with diarrhoea caused by enteroviruses circulating in Guangdong Province in 2009-2012 had mild or moderate gastrointestinal symptoms. Although enterovirus-related diarrhoea caused additional multi-organ dysfunction syndrome, hepatic injury and myocardial injury in children with HFMD/HA, timely intervention efficiently reduced disease severity and improved outcome.
\end{abstract}

Keywords: Enterovirus, Diarrhoea, Hand, Foot and mouth disease, Herpangina, Clinical features

\footnotetext{
*Correspondence: apollo_zhou@126.com; yunanzhujiang@163.com

${ }^{1}$ Laboratory of Emerging Infectious Diseases and Division of Laboratory

Medicine, Zhujiang Hospital, Southern Medical University, No. 253 Gong Ye

Da Dao Zhong, Guangzhou 510282, China

Full list of author information is available at the end of the article
} 


\section{Background}

Enteroviruses (EVs) are known to cause hand, foot and mouth disease/herpangina (HFMD/HA), with numerous epidemics worldwide, including China [1]. Several complications have been reported in HFMD/HA, including meningitis, encephalitis, acute flaccid paralysis, acute cardiopulmonary failure, respiratory infection, myocardial injury, and diarrhoea $[2,3]$. Among these complications in HFMD/HA, diarrhoea is less prominent because of its low prevalence and generally self-limiting nature. In China, diarrhoea has generally been neglected or only mentioned in most studies of HFMD/HA. Moreover, EV detection is often not included in pathogen surveillance of diarrhoea; thus, little information on the epidemiology and clinical features of enterovirus-related diarrhoea is available in China. Diarrhoea can cause various degrees of illness, ranging from self-limited changes in stool consistency to life-threatening complications and even death. Diarrhoea deserves attention because it is the third leading cause of death in young children, claiming millions of lives annually worldwide [4].

Considering the global presence of HFMD/HA, there is an urgent need to elucidate the clinical characteristics of enterovirus-related diarrhoea and its impact on HFMD/ HA. Therefore, the purpose of this study was to describe the clinical features and implications of enterovirus-related diarrhoea in children with HFMD/HA admitted to Zhujiang Hospital during 2009-2012.

\section{Methods}

\section{Study population and data collection}

All cases were inpatients admitted to the Paediatric Department of Zhujiang Hospital between January 2009 and December 2012. Zhujiang Hospital is an officially designated tertiary hospital for treatment of HFMD in Guangzhou City (capital of Guangdong Province, China) and its neighbouring districts. Anonymous processing of patient information ensured the privacy of patients, and the study was approved by the Ethics Committee of Zhujiang Hospital (Ratification No: ZJYY-2013-YXJYZX001). Signed informed consent was obtained from parents or guardians. Cases were included if they met all of the inclusion criteria and none of the exclusion criteria. Inclusion criteria were: paediatric inpatients with eruptions or fever; and real-time fluorescence reverse transcriptase polymerase chain reaction (RT-PCR) evidence of positive enterovirus. Exclusion criteria were: medical history of neurological disease, congenital heart disease or immune deficiency; or evidence of infection by rotavirus, norovirus, Shigella, Campylobacter or Salmonella species. Clinical data for each case were retrospectively reviewed and extracted from medical records, and patients were assigned to the diarrhoea or no diarrhoea group, depending on whether they presented with diarrhoea. Clinical features were compared between the two groups to explore the implications of diarrhoea in HFMD/HA.

\section{Stool sample collection and testing}

Stool specimens from each case were collected for extraction of RNA and stored at $-80{ }^{\circ} \mathrm{C}$. Viral RNAs were extracted from $140-\mu$ l stool samples using the QIAamp Viral RNA Mini Kit system (Qiagen, Hilden, Germany). Extracted RNAs were used for real-time fluorescence RT-PCR and/or the amplification of partial-length fragments of enterovirus viral protein (VP)1/5'-untranslated region (UTR). Serotypes of enterovirus 71 (EV71) and coxsackievirus 16 (CA16) were jointly determined by enteroviruses kit, EV71 kit, and CA16 kit (Shanghai ZJ Bio-Tech Co. Ltd., China). The other serotypes were determined by sequencing partial-length fragments of VP1/5'-UTR, as described previously $[5,6]$. The enterovirus serotype was ascertained by performing BLAST analysis along with EV sequences in GenBank. Each patient with diarrhoea received routine stool examinations, and samples were tested for bacterial cultures of Shigella, Campylobacter and Salmonella species. Routine stool examinations include assessment of colour, consistency, parasite ova, white blood cells, red blood cells, occult blood, transferrin, and rotavirus and norovirus antigens.

\section{Clinical definitions}

Diarrhoea was defined as a change in stool consistency, or defecation $\geq 3$ times/day. The severity of diarrhoea was assessed by a scoring system based on vomiting, frequency and duration of diarrhoea, electrolyte disturbance, dehydration status, and fever. A total score $>11$ was classified as serious diarrhoea, and $\leq 11$ as mild diarrhoea [7]. Central nervous system (CNS) involvement referred to the presence of two or more of the following: vomiting, irritability, myoclonic jerk, convulsion, changed muscular tension, decreased tendon reflex, headache, and neck stiffness. Patients who also had altered consciousness, paralysis, or CNS lesions on neuroimaging were considered to have severe CNS involvement. Acute respiratory infection (ARI) was diagnosed by the presence of rhinorrhoea and/or cough, and patients with pneumonia or bronchopneumonia were judged to have severe (ARI). Myocardial injury was diagnosed based on laboratory evidence of elevated cTnI, or elevated CK$\mathrm{MB}$ plus $\mathrm{CK}-\mathrm{MB} / \mathrm{CK}>0.25$. cTnI $>0.04 \mu \mathrm{g} / \mathrm{l}$, ratio of $\mathrm{CK}-\mathrm{MB} / \mathrm{CK}>0.25$ and $\mathrm{CK}-\mathrm{MB}>44 \mathrm{U} / \mathrm{L}(2 \mathrm{~m}-36 \mathrm{~m})$, CK-MB > $37 \mathrm{U} / \mathrm{L}(37 \mathrm{~m}-72 \mathrm{~m})$, or CK-MB > $31 \mathrm{U} / \mathrm{L}$ $(>73 \mathrm{~m})$ were considered elevated. Cases with alanine aminotransferase $>40 \mathrm{U} / \mathrm{l}$ were considered to have hepatic injury. 


\section{Statistical analysis}

Statistical analysis was performed by SPSS version 13.0 (SPSS, Chicago, IL, USA). Differences between two groups of continuous variables were analysed by the independent samples $t$-test, whereas those of categorical variables were analysed by the $\chi^{2}$ test. All $p$ values were two-sided, and $p<0.05$ was considered statistically significant.

\section{Results}

A total of 1778 EV-related HFMD/HA patients were admitted to the Paediatric Department of Zhujiang Hospital during 2009-2012. There were 872 inpatients and 896 outpatients. Most patients with HFMD/HA were admitted in April to July, with a peak between May and June (Fig. 1). There was a shift in the dominant enterovirus serotype during 2009-2012: CA16 in 2009, EV71 in 2010-2011, and other EV serotypes in 2012 (Fig. 1). There was a low prevalence of enterovirusrelated diarrhoea and varied prevalence of different EV serotypes. Of the 872 inpatients, 804 were eligible for inclusion in this study, and 28 presented with diarrhoea (3.5\%). We identified enterovirus serotypes in 767 cases, and the detailed distribution of the serotypes in the no diarrhoea and diarrhoea groups is shown in Fig. 2a and b, respectively. Diarrhoea prevalences of different EV serotypes varied considerably: Echo 14 (100 \%), CVA21 (100 \%), Echo 2 (50.0 \%), CVA9 (33.3\%), CVA10 (9.7 \%), CVA4 (6.3 \%), CVA6 (4.5\%), EV71 (2.3 \%) and CVA16 (0.6 \%).

\section{Severity and gastrointestinal symptoms of enterovirus-related diarrhoea}

Based on the scoring system, the 28 cases of diarrhoea had a score of $4-17$, and 23 cases $(82.1 \%)$ scored $\leq 11$. Table 1 shows that most patients with enterovirus-related diarrhoea had mild gastrointestinal symptoms, as characterised by high prevalence of no dehydration (82.1\%), short duration of diarrhoea $(78.6 \%)$ and watery stools (75.0 \%). Two patient with diarrhoea caused by EV71 died: one died from cardiopulmonary failure, and the other from multi-organ dysfunction syndrome (MODS).

\section{Effect of enterovirus-related diarrhoea on outcomes and severity of HFMD/HA}

There were no significant differences between the diarrhoea and no diarrhoea groups for duration of hospitalisation and prevalence of death, altered consciousness, paralysis, CNS involvement, severe CNS involvement, ARI and severe ARI (Table 2). Compared with the no diarrhoea group, the diarrhoea group had a significantly higher prevalence of MODS (10.7 vs $0.40 \%)(p=0.001)$, hepatic injury (14.3 vs $3.4 \%)(p=0.019)$, myocardial injury (21.4 vs $6.1 \%)(p=0.002)$ and convulsion $(21.4$ vs $7.2 \%)(p=0.016)$.

\section{Discussion}

Our study demonstrated that most cases of enterovirusrelated diarrhoea $(82.1 \%)$ were mild without severe gastrointestinal symptoms. Among the 28 cases of diarrhoea, there was a low prevalence of severe dehydration (14.3\%), frequent episodes of diarrhoea ( $\geq 6 /$ day) (35.7 \%), long duration of diarrhoea ( $\geq 6$ days) (21.4\%), visible bloody stool $(0 \%)$, frequent vomiting ( $\geq 5$ episodes/day) $(10.7 \%)$ and long duration of vomiting ( $\geq 3$ days) $(10.7 \%)$. Our study also showed a low prevalence $(3.5 \%)$ of diarrhoea caused by enteroviruses circulating in Guangdong during 2009-2012. Although enteroviruses are not well known for causing diarrhoea, some studies have shown them to be causative agents [8-12]. Some other members of the Picornaviridae, such as Aichi virus, parechovirus, and bocavirus, have been recently associated with diarrhoea; however, only with a small number of cases. Therefore, there was only a small probability that any of the cases of

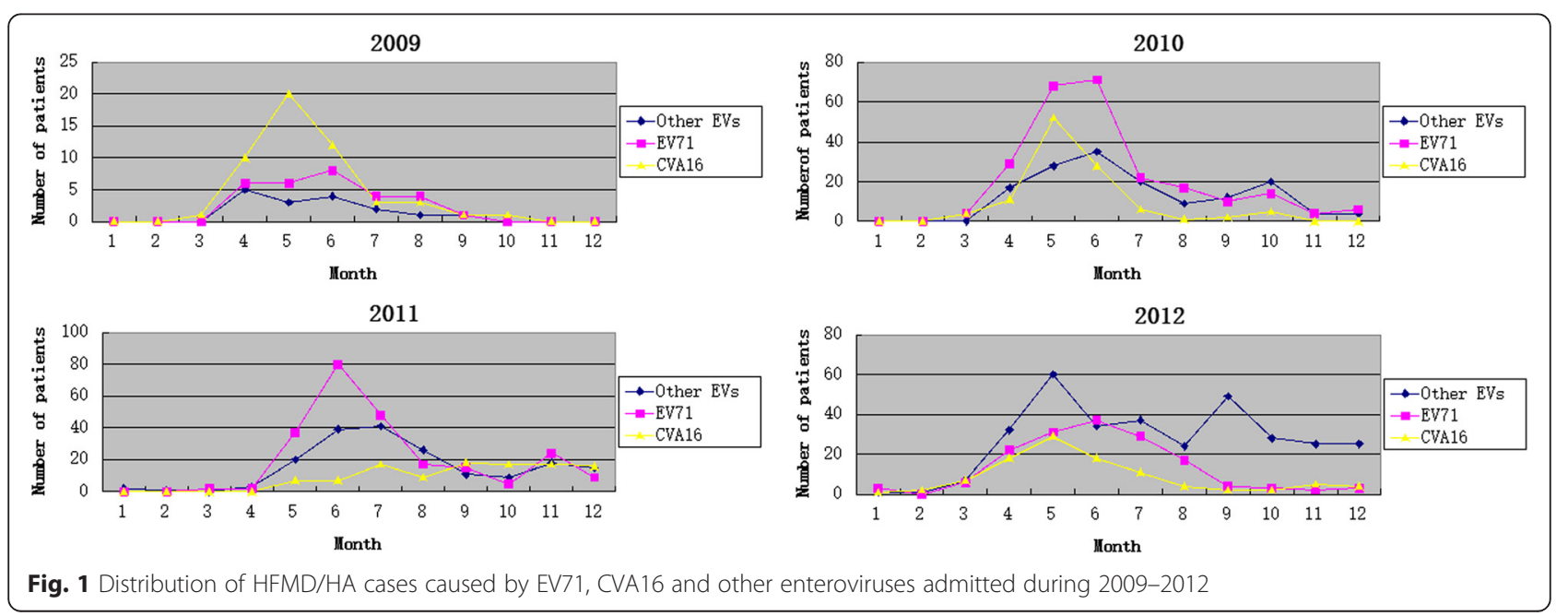




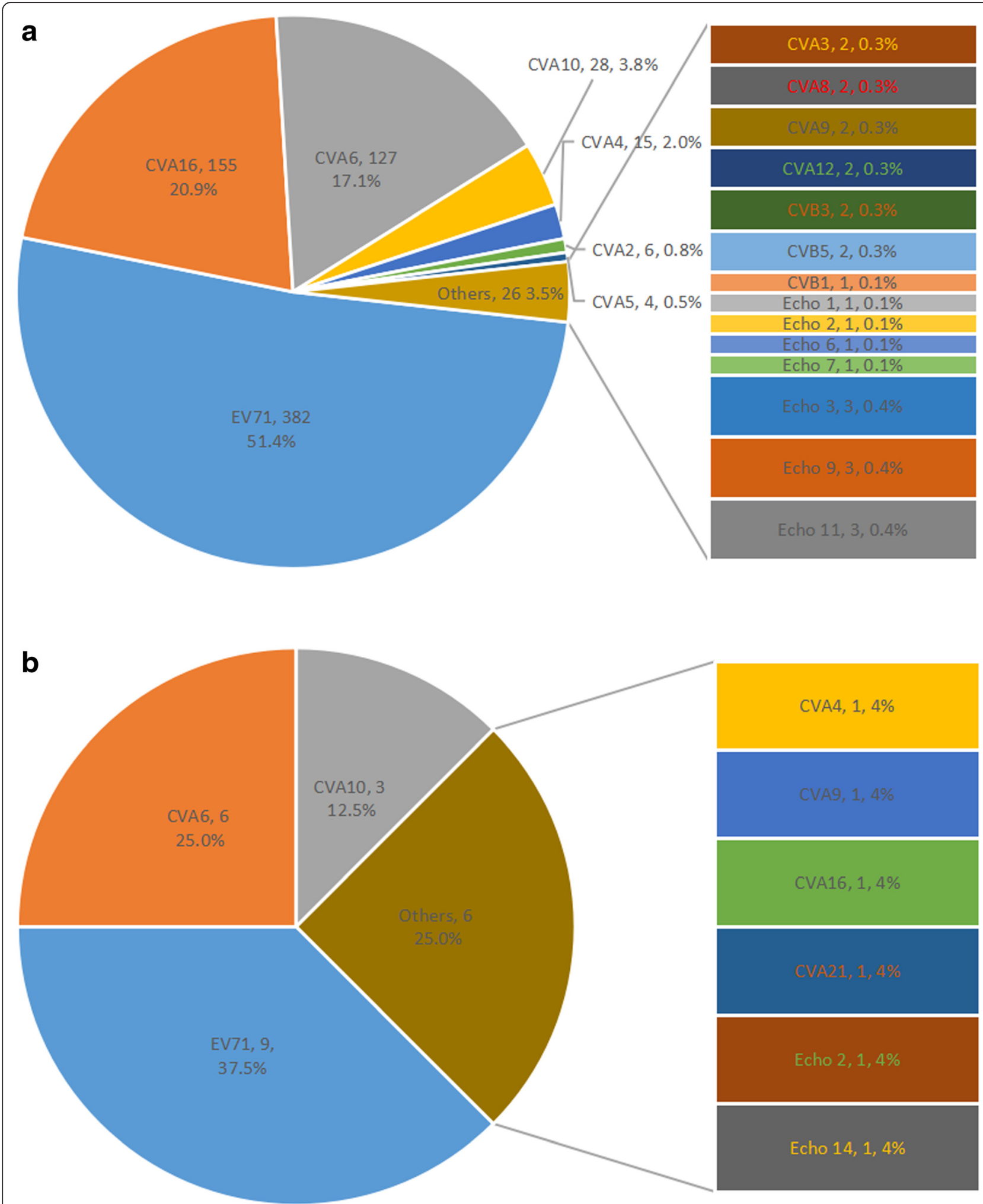

Fig. 2 a Constituent ratios of EV serotypes in group without diarrhoea. b Constituent ratios of EV serotypes in group with diarrhoea 
Table 1 Clinical presentations of 28 enterovirus-related HFMD/HA inpatients with diarrhoea

\begin{tabular}{|c|c|}
\hline Characters & $N(\%)$ \\
\hline Age (months) & $28.7 \pm 17.7$ \\
\hline \multicolumn{2}{|l|}{ Duration of diarrhoea (days) } \\
\hline $1-4$ & $22(78.6)$ \\
\hline 5 & $0(0)$ \\
\hline$\geq 6$ & $6(21.4)$ \\
\hline \multicolumn{2}{|c|}{ Maximum no. diarrhoeal stools/24 h } \\
\hline$\leq 3$ & $10(35.7)$ \\
\hline $4-5$ & $8(28.6)$ \\
\hline$\geq 6$ & $10(35.7)$ \\
\hline Vomiting & $11(39.3)$ \\
\hline \multicolumn{2}{|l|}{ Duration of vomiting (days) } \\
\hline 0 & $17(60.7)$ \\
\hline 1 & $7(25.0)$ \\
\hline 2 & $1(3.6)$ \\
\hline$\geq 3$ & $3(10.7)$ \\
\hline \multicolumn{2}{|c|}{ Maximum no. vomiting episodes/24 h } \\
\hline 0 & $17(60.7)$ \\
\hline 1 & $0(0.0)$ \\
\hline $2-4$ & $8(28.6)$ \\
\hline$\geq 5$ & $3(10.7)$ \\
\hline \multicolumn{2}{|l|}{ Maximum body temperature $\left({ }^{\circ} \mathrm{C}\right)$} \\
\hline$<37.1$ & $1(3.6)$ \\
\hline $37.1-38.4$ & $2(7.1)$ \\
\hline $38.5-38.9$ & $9(32.1)$ \\
\hline$\geq 39$ & $16(57.1)$ \\
\hline \multicolumn{2}{|l|}{ Dehydration } \\
\hline None & $23(82.1)$ \\
\hline Mild & $1(3.6)$ \\
\hline Severe & $4(14.3)$ \\
\hline \multicolumn{2}{|l|}{ Stool consistency } \\
\hline Watery & $21(75.0)$ \\
\hline Mushy & $6(21.4)$ \\
\hline Mucous & $1(3.6)$ \\
\hline Occult blood in stool & $4(14.3)$ \\
\hline Mild diarrhoea (score $\leq 11)$ & $23(82.1)$ \\
\hline Severe diarrhoea (score > 11) & $5(17.9)$ \\
\hline
\end{tabular}

diarrhoea in the present study were caused by coinfection with these viruses and enteroviruses. Rotavirus, norovirus, and Shigella have been reported as major causative pathogens of diarrhoea in Guangdong Province and its neighbouring districts [13-16]. Although we did not perform comprehensive tests to exclude all possible cofactors of diarrhoea, it is reasonable to suggest that enteroviruses were responsible for most of the cases since they were all negative for major potential diarrhoearelated pathogens.

Enteroviruses are pathogens that can invade and reproduce in intestinal cells and induce severe inflammation in human intestinal epithelial cells [17-19]. According to the pathogenic mechanisms proposed by Navaneethan and Giannella, enterovirus-related diarrhoea is initiated by inflammation involving activation of cytokines and inflammatory mediators [20]. EV71 and CVA6 were the two serotypes that claimed more than half of diarrhoea cases (15/28), the diarrhoea prevalences of different EV serotypes varied considerably: Echo 14 (100\%), CVA21 (100 \%), Echo 2 (50.0 \%), CVA9 (33.3\%), CVA10 (9.7\%), CVA4 (6.3\%), CVA6 (4.5\%), EV71 (2.3 \%) and CVA16 (0.6 \%). Although no particular serotype of enterovirus is especially associated with diarrhoea, previous studies have suggested that some serotypes of echovirus and coxsackievirus B are more likely to cause diarrhoea [10-12, 21, 22], which is similar to our results. Enteroviruses are generally not listed as potential pathogens for diarrhoea, which leads to limited information about enterovirus-related diarrhoea, and aetiological misdiagnosis. Therefore, physicians should consider enteroviruses as potential pathogens when treating diarrhoea, especially for cases admitted during times of seasonal enterovirus circulation. Moreover, more studies should be carried out to elucidate the discrepancies and clinical features of diarrhea caused by different serotypes of enterovirus due to the extreme lack of such knowledge.

Enterovirus-related diarrhoea did not did not exert substantial influence on the severity of HFMD/HA in patients who received appropriate and timely medical intervention. No difference was observed between the diarrhoea and no diarrhoea groups regarding duration of hospitalisation and prevalence of death, CNS involvement, severe CNS involvement, paralysis, altered consciousness, ARI and severe ARI. Although the diarrhoea group had significantly higher prevalence of MODS (10.7 vs $0.40 \%)$, hepatic injury (14.3 vs $3.4 \%$ ), myocardial injury (21.4 vs $6.1 \%$ ) and convulsion (21.4 vs 7.2 ) than no diarrhoea group, all of these manifestations were short term and reversible with treatment. The limited influence of diarrhoea on severity of HFMD/HA was largely due to the following factors: the mild or moderate nature of most diarrhoea, without severe and long-term dehydration; appropriate and timely medical intervention; the inflammatory mechanism of enterovirus-related diarrhoea; and inability of enterovirus particles and proinflammatory cytokines to cross the blood-brain barrier in most conditions. Evasion and modulation of the gastrointestinal defence systems of the host are essential for pathogens causing infectious diarrhoea [20]. Thus, it is reasonable to assume that higher levels of cytokines and inflammatory mediators will be produced in HFMD/HA patients with 
Table 2 Comparisons of clinical features between patients with and without diarrhoea

\begin{tabular}{llll}
\hline Characteristics & Diarrhoea (\%) $(n=28)$ & No diarrhoea $(\%)(n=776)$ & \\
\hline Demographic & & $29.1 \pm 17.6$ & 0.015 \\
$\quad$ Diarrhoea (months) & $18.3 \pm 21.2$ & $2.00: 1$ & $459(59.1)$ \\
$\quad$ Male-to-female ratio & $1.55: 1$ & $446(57.5)$ & 0.506 \\
Fever $\geq 39{ }^{\circ} \mathrm{C}$ & $16(57.1)$ & $23 / 675(3.4)$ & 0.832 \\
Fever duration $\geq 3$ days & $20(71.4)$ & $30 / 703(6.1)$ & 0.142 \\
Elevated ALT & $4(14.3)$ & $56(7.2)$ & 0.019 \\
Elevated myocardial enzyme & $6(21.4)$ & $65(8.5)$ & 0.002 \\
Convulsion & $6(21.4)$ & $86(11.1)$ & 0.016 \\
Altered consciousness & $4(14.3)$ & $233(30.0)$ & 0.291 \\
Severe CNS involvement & $4(14.3)$ & $99(12.8)$ & 0.543 \\
CNS involvement & $12(42.9)$ & $244(31.4)$ & 0.147 \\
Severe ARI & $5(17.9)$ & $3(0.40)$ & 0.393 \\
ARI & $11(39.3)$ & $28(3.6)$ & 0.410 \\
MODS & $3(10.7)$ & $12(1.5)$ & 0.001 \\
Paralysis & $0(0.0)$ & $6.3 \pm 4.2$ & 0.619 \\
Mortality & $2(7.1)$ & $6.0 \pm 2.7$ & 0.082 \\
Duration of hospitalization (days) & 0.792 \\
\hline
\end{tabular}

Note. $A L T$ alanine aminotransferase, $A R I$ acute respiratory infection, CNS central nervous system, MODS multi-organ dysfunction syndrome

than without diarrhoea. These higher levels can cause increased prevalence of hepatic injury, myocardial injury and MODS in HFMD/HA patients with diarrhoea. In our study, most cases of diarrhoea $(82.1 \%)$ presented without dehydration, and timely hospital treatment abolished dehydration when it did occur. Therefore, it was inflammation rather than haemodynamic disturbance caused by dehydration that was responsible for the differences between the diarrhoea and no diarrhoea groups. Because MODS was initiated by inflammation, there was no significant increase in mortality in the diarrhoea group when patients received timely medical intervention. The exceptionally high prevalence of convulsion in the diarrhoea group was largely because convulsion, unlike other neurological manifestations, can be induced in young children by mere dehydration or electrolyte imbalance. The absence of any substantial increase in prevalence of CNS involvement, severe CNS involvement and paralysis in HFMD/HA with diarrhoea was a relief for such patients.

Some limitations may exist because our study was a retrospective observation based on the enterovirus serotypes that were circulating in Guangdong province during 2009-2012. Moreover, the limited number of diarrhoea cases and other possible co-infections may also have biased the results. Intensive treatment administered during hospitalization also limited the natural progression of diarrhoea and even prevented its impact on the severity of HFMD/HA. Therefore, more studies should be carried out to elucidate the characteristics of enterovirus-related diarrhoea and their implications in HFMD/HA.

\section{Conclusions}

From 2009 to 2012, patients with diarrhoea caused by circulating enteroviruses had mostly mild or moderate gastrointestinal symptoms. Timely intervention reduced disease severity and improved outcome in children with HFMD/HA. As a result of its mild severity and being initiated by inflammation, enterovirus-related diarrhoea did not exert a major influence on severity or outcome in HFMD/HA inpatients in Guangdong, China, during 2009-2012.

\section{Products}

Pan-enteroviruses kit, EV71 kit, CA16 kit (Shanghai ZJ Bio-tech Co., LTD); ViiA ${ }^{\text {si }} 7$ system (Life Technologies, USA); virus transport system (Copan Italia S.P.A, Italy); QIAamp Viral RNA Mini Kit system (Qiagen, Hilden, Germany).

\section{Abbreviations}

ALT: alanine aminotransferase; ARl: acute respiratory infection; AST: aspartate aminotransferase; CK: creatine kinase; CK-MB: creatine kinase-MB; CNS: central nervous system; CVA: coxsackievirus A; Echo: echovirus; HFMD/HA: hand, foot and mouth disease/herpangina; MODS: multi-organ dysfunction syndrome; RT-PCR: reverse transcriptase polymerase chain reaction; UTR: untranslated region.

\section{Competing interests}

The authors declare that they have no competing interests. No competing interests exists in this study due to commercial or other associations (e.g., pharmaceutical stock ownership, consultancy, advisory board membership, relevant patents, or research funding). No competing interests exists in the submission of this manuscript, and the manuscript is approved by all of the authors for publication, I declare on behalf of my co-authors that the work described was original research that has not been published previously and is not under consideration for publication elsewhere in whole or in part. 


\section{Authors' contributions}

H-TZ carried out the data collection for clinical investigation and laboratory evaluation, performed genetic serotype identification, and drafted the initial manuscript. H-SY participated in data collection and analysis and revised the manuscript. NY performed enterovirus testing with RT-PCR, and reviewed and revised the manuscript. Y-HG participated in serotype identification. Y-XP performed enterovirus isolation and serotype identification using RT-PCR. S-HT confirmed all of the cases enrolled in this study and jointly carried out the initial analyses on clinical features, especially on neurological manifestations. $\mathrm{M}-\mathrm{JC}$ performed clinical specimen collection and virus isolation and identification. BW analysed the clinical features of cases enrolled in this study. MY participated in data collection. All of the authors gave final approval of the version to be published.

\section{Acknowledgements}

We thank Fu Ning (Department of Immunology, School of Basic Medical Sciences, Southern Medical University) for her advice and revision of the manuscript; all of the doctors in the Department of Paediatrics of Zhujiang Hospital for providing medical records of inpatients; Xia N (National Institute of Diagnostics and Vaccine Development in Infectious Disease, Xiamen University) for providing the primers for amplifying the partial-length fragments of the 5'-UTR of enterovirus. The work was supported by the National Projects of Major Infectious Disease Control and Prevention of China (grant number 2012ZX10004213) and the National High Technology Research and Development Program of China (863 Program; grant number 2011AA02A116)

\section{Author details}

'Laboratory of Emerging Infectious Diseases and Division of Laboratory Medicine, Zhujiang Hospital, Southern Medical University, No. 253 Gong Ye Da Dao Zhong, Guangzhou 510282, China. 'Department of Pediatrics, Zhujiang Hospital, Southern Medical University, Guangdong 510282, China. ${ }^{3}$ Department of Clinical Laboratory, Hainan Provincial People's Hospital, No.

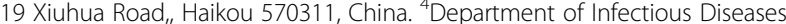
Zhujiang Hospital, Southern Medical University, Guangdong 510282, China.

Received: 26 March 2015 Accepted: 10 March 2016

\section{Published online: 16 March 2016}

\section{References}

1. National statutory report of incidence and death statistics of infectious disease. http://www.moh.gov.cn/publicfiles//business/htmlfiles/wsb/pyqxx/list.htm.

2. Hsiung GD, Wang JR. Enterovirus infections with special reference to enterovirus 71. J Microbiol Immunol Infect. 2000;33:1-8.

3. Stalkup JR, Chilukuri S. Enterovirus infections: a review of clinical presentation, diagnosis, and treatment. Dermatol Clin. 2002;20:217-23.

4. Distribution of causes of death among children aged $<5$ years. http://apps. who.int/gho/data/view.main.CMDRWORLD?lang=en.

5. Nix WA, Oberste MS, Pallansch MA. Sensitive, seminested PCR amplification of VP1 sequences for direct identification of all enterovirus serotypes from original clinical specimens. J Clin Microbiol. 2006;44:2698-704.

6. Ge S, Yan $\mathrm{Q}, \mathrm{He} \mathrm{S}$, et al. Specific primer amplification of the VP1 region directed by $5^{\prime}$ UTR sequence analysis: enterovirus testing and identification in clinical samples from hand-foot-and-mouth disease patients. J Virol Methods. 2013;193:463-9.

7. Ruuska T, Vesikari T. Rotavirus disease in Finnish children: use of numerical scores for clinical severity of diarrhoeal episodes. Scand J Infect Dis. 1990;22 259-67.

8. Chaimongkol N, Khamrin P, Suantai B, et al. A wide variety of diarrhea viruses circulating in pediatric patients in Thailand. Clin Lab. 2012;58:117-23.

9. Maslin J, Kohli E, Leveque $\mathrm{N}$, et al. Characterisation of viral agents with potential to cause diarrhea in Djibouti. Med Trop (Mars). 2007;67:249-55.

10. Rao DC, Ananda Babu M, Raghavendra A, et al. Non-polio enteroviruses and their association with acute diarrhea in children in India. Infect Genet Evol. 2013;17:153-61

11. Abe $\mathrm{O}$, Kimura $\mathrm{H}$, Minakami $\mathrm{H}$, et al. Outbreak of gastroenteritis caused by echovirus type 6 in an orphanage in Japan. J Infect. 2000;41:285-6.

12. Oishi I, Maeda A, Otsu K, et al. An outbreak of diarrhea in school children associated with Coxsackie virus B-3 infection. Biken J. 1979;22:21-4.

13. Li CS, Chan PK, Tang JW. Prevalence of diarrhea viruses in hospitalized children in Hong Kong in 2008. J Med Virol. 2009;81:1903-11.
14. Zeng M, Chen J, Gong ST, et al. Epidemiological surveillance of norovirus and rotavirus diarrhea among outpatient children in five metropolitan cities. Zhonghua Er Ke Za Zhi. 2010;48:564-70.

15. Lee MK, Chan PK, Ho II, Lai WM. Enterovirus infection among patients admitted to hospital in Hong Kong in 2010: epidemiology, clinical characteristics, and importance of molecular diagnosis. J Med Virol. 2013;85:1811-7.

16. Li DD, Li H, Guo RN, et al. Pathogens of high incidence of other infectious diarrhea in Guangdong Province, from November 2008 to January 2009. Bing Du Xue Bao. 2010;26:150-2.

17. Xu J, Yang Y, Wang C, Jiang B. Rotavirus and coxsackievirus infection activated different profiles of toll-like receptors and chemokines in intestinal epithelial cells. Inflamm Res. 2009;58:585-92.

18. Zhang YC, Jiang SW, Gu WZ, et al. Clinicopathologic features and molecular analysis of enterovirus 71 infection: report of an autopsy case from the epidemic of hand, foot and mouth disease in China. Pathol Int. 2012:62:565-70.

19. Chi C, Sun Q, Wang S, et al. Robust antiviral responses to enterovirus 71 infection in human intestinalepithelial cells. Virus Res. 2013;176:53-60.

20. Navaneethan U, Giannella RA. Mechanisms of infectious diarrhea. Nat Clin Pract Gastroenterol Hepatol. 2008;5:637-47.

21. Carvalho RP, Kirchner E, Moura RA, Murahovschi J. Acute gastroenteritis associated with coxsackie B-6 virus in infants and children, in Sao Paulo, Brazil. Rev Inst Med Trop Sao Paulo. 1977;19:73-6.

22. Tai FH, Wang HC. Acute gastroenteritis associated with echovirus type 1. Southeast Asian J Trop Med Public Health. 1973:4:311-5.

\section{Submit your next manuscript to BioMed Central and we will help you at every step:}

- We accept pre-submission inquiries

- Our selector tool helps you to find the most relevant journal

- We provide round the clock customer support

- Convenient online submission

- Thorough peer review

- Inclusion in PubMed and all major indexing services

- Maximum visibility for your research

Submit your manuscript at www.biomedcentral.com/submit
Biomed Central 